\title{
Judder-Induced Edge Flicker at Zero Spatial Contrast
}

\author{
James Larimer ${ }^{1}$, Christine Feng ${ }^{2}$, Jennifer Gille ${ }^{3}$, Victor Cheung ${ }^{4}$ \\ ${ }^{1}$ NASA, ${ }^{2}$ Princeton University, ${ }^{3}$ Raytheon ITSS, ${ }^{4}$ San Jose State University \\ Ames Research Center, Moffett Field, CA 94035-1000
}

\begin{abstract}
Judder is a motion artifact that degrades the quality of video imagery. Smooth motion appears jerky and can appear to flicker along the leading and trailing edge of the moving object. In a previous paper (1) we demonstrated that the strength of the edge flicker signal depended upon the brightness of the scene and the contrast of the moving object relative to the background. Reducing the contrast between foreground and background reduced the flicker signal. In this report, we show that the contrast signal required for judder-induced edge flicker is due to temporal contrast and not simply to spatial contrast. Bars made of random dots of the same dot density as the background exhibit edge flicker when moved at sufficient rate.
\end{abstract}

\section{Introduction}

Objects in nature tend to move in smooth trajectories. When motion is sampled by a video camera and reconstructed on a display that natural smooth motion can appear to be jerky and irregular. This visual artifact is called motion judder. Motion judder is often associated with frame rate conversion, for example, converting from cinema to video frame rates (2), but this is not the only source of this artifact. When the sampling frequency is low, $24 \mathrm{~Hz}$ or less, and the object motion velocity is fast the reconstructed motion sequence can add an annoying visual artifact that appears as flicker along the leading and trailing edge of the moving object. This artifact is common in cinema and television. We have called this artifact judder-induced edge flicker (1).

Judder and judder-induced edge flicker are caused by the discrete sampling of time and the resulting quantization of the continuous spatial-temporal variation generated by natural motion. The sampling process converts continuous motion into a series of discrete static images that are reconstructed and viewed seriatim when the video sequence is reproduced on a display device.

When the eye is presented with a moving object it tends to pursue the moving object with smooth pursuit eye movements that lock onto the velocity of the moving object in the scene frame of reference. The pursuit eye movements attempt to match the object velocity thus stabilizing the moving object's image at a fixed location on the retina. Pursuit eye movements are effectively a continuous coordinate system translation, remapping the origin, a fixed point on the retina, continuously in time.

The reconstruction of continuous motion via a discrete quantization process results in a series of momentarily static images in world coordinates. The moving object in world coordinates jumps across the frame of reference in discrete static steps. During the reconstruction the eye is smoothly pursuing the motion across the frame of reference. The pursuit eye movements match the average velocity of the moving object in nature. The reconstructed object, however, only moves relative to the scene at the sampling rate, i.e., when the image changes. During the brief time a sample is being reconstructed on the display, there is no relative motion.

The eye continues to move during the static periods, so the image of the moving object on the retina moves in the opposite direction to the smooth pursuit motion on the retina. 
This periodic slip signal, i.e., at the frame rate of the reconstruction, produces a flicker at the edge of the object as it is imaged onto the observer's retina. This is perceived as flicker when the contrast, brightness and motion rates together exceed a threshold value. Our experiment measures this threshold in units of object motion quantified as pixels per second. To see this edge flicker the slip rate must exceed the threshold for seeing temporal change on the retina.

In this experiment we document that it is only the slip rate that matters. We do this by showing that a target of moving bars made of random dots on a background of random dots of the same dot density as the bars also produces judder-induced edge flicker. Random dot bars on a random dot background of the same dot density have no spatial contrast relative to the background. When the bars stop moving, they are invisible on the background. They can only be perceived as bars when they are moving. It is the coherent motion of the dots that produces the edge signal defined by the spatially coherent motion of a set of random dots that evokes the perception of moving bars, this signal is also sufficient to produce judder and judder-induced edge flicker.

\section{Experiment}

Subjects: Three subjects, all authors of the paper, participated in the experiment. All had normal vision or normal vision after eyeglass optical correction. One observer was an experienced participant in psychophysical experiments; the other two were novice observers.

Stimuli: Three bar patterns were used in this experiment. The stimuli were all moving bar gratings at a spatial frequency of approximately 1 cycle per degree. The bars were approximately $4 \mathrm{~mm}$ wide and $16 \mathrm{~mm}$ high \& spaced $16 \mathrm{~mm}$ apart. When viewed at a distance of approximately $800 \mathrm{~mm}$ from the screen each bar subtends about $0.25^{\circ}$ in width and $1^{\circ}$ in height and repeats once every $1^{\circ}$. The window in which the bars appeared was apodized so the bars moving from left to right appear to fade in, move across the screen, and fade out on the right hand side of the presentation aperture. The presentation aperture was $80 \mathrm{~mm}$ by $16 \mathrm{~mm}$ on the screen. The aperture edge apodization was designed to reduce any aperture edge effects that might be present in the targets. All targets were presented on a CRT display.

Three bar types were used. One set of bars consisted of solid black bars on a white background. One set consisted of random dot bars where $75 \%$ of the pixels in the bar area was black and $25 \%$ white. For these bars, the reverse ratio of black and white pixels was used to produce the background. Finally, a third set of patterns were constructed where half of all of the pixels on the screen were randomly decided to be either white or black irrespective of whether or not the pixel was in a bar or background region of the image. These bars could only be perceived when the bars were moving; otherwise the aperture appeared to be an $80 \times 16 \mathrm{~mm}$ aperture of apodized random dots. The bars were viewed at three brightness levels controlled by neutral density filters.

Sampling Rates \& Reconstruction: The smooth natural motion was sampled at 15 $\mathrm{Hz}$ using an MatLab algorithm designed to emulate the discrete sampling of continuous real object motion by a film based motion picture camera sampling at a frequency of $15 \mathrm{~Hz}$. The sampling period was set to be instantaneous, thus there was no motion blur in these images. The sequence was reproduced on a CRT running at a frame rate of $75 \mathrm{~Hz}$ in progressive scan mode under computer control. To reconstruct the $15 \mathrm{~Hz}$ sampling, each sample frame was repeated 5 times at $75 \mathrm{~Hz}$. This is the same method used in film projectors to eliminate the perception of full field flicker.

A second reconstruction scheme was employed where the $15 \mathrm{~Hz}$ sampled sequence was up-sampled to $75 \mathrm{~Hz}$ using a fade filter to produce the 4 inner frames 
between sampled, i.e., corner frames. The inner frames were convex sums of the bounding corner frame images of various proportions linear in time. This produces a smooth but nonetheless discrete fading from one corner frame to the next. We have called this filter the "Movieola" filter after the film editing apparatus, the Movieola, used to edit motion picture film. A movieola optically generates a fade from one frame to the next.

Procedure: The subject's task was to adjust the motion rate of the bars in pixels/sec until the leading and trailing edges of the bars just appeared to flicker. Each subject made three settings at each condition within a session. Sessions were repeated over days.

Results: The results are shown in Figures 1 $\& 2$ below.
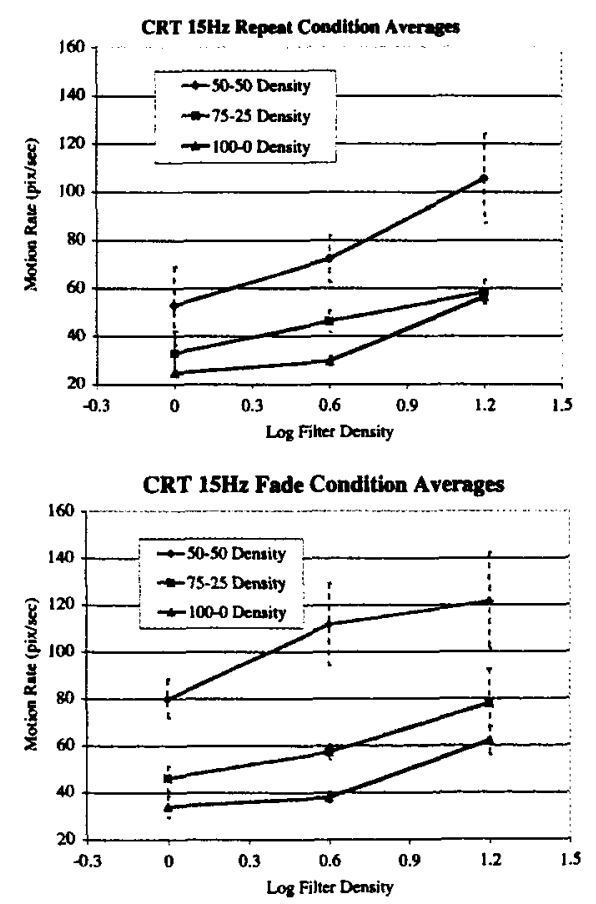

Fig. $1 \& 2$. The figures show a plot of the threshold motion rates as a function of three brightness levels from approximately 35 $\mathrm{cd} / \mathrm{m}^{2}, 8.75 \mathrm{~cd} / \mathrm{m}^{2}, \& 2.18 \mathrm{~cd} / \mathrm{m}^{2}$ at three different bar contrasts. On the top are the results for up-sampling by replication and on the bottom for up-sampling by Movieola filter.

\section{Conclusion}

The results confirm that decreasing contrast or brightness increases the motion rate threshold to perceive judder-induced edge flicker. Additionally, even a condition where the contrast signal only exists when the bars are moving, i.e., there is no brightness difference between the foreground and background, produces judder-induced edge flicker.

These results show that motion artifacts are determined by sampling rates and the rate at which the image sequence is up-sampled during reconstruction. A very simple and easily implemented filter mitigates this annoying visual artifact. Because highresolution displays require severe data rates, finding the sweet spot for sampling and reconstruction will have important consequences for electronic displays and image communications systems. This work is designed to investigate the lower limits of sampling and some simple up-sampling schemes that may enable low power highresolution imagery in the future.

\section{References}

(1) Larimer, J., Gille, J., Wong, J. (2001) Judder-induced edge flicker in moving objects. SID Digest 2001, pp. 10941098.

(2) Sonehara, H., Nojiri, Y., Iguchi, K.,Sugiura, Y., Hirabayashi, H. (1996) Reduction of Motion Judder on Video Images Converted from Film, SMPTE Tech. Conf. World Media Expo, 138-1.; NHK Lab Note \#450, http://www.nhk. or.jp/strl /publica/labnote/lab450.html; Philips Semiconductors, http://www-us. semiconductors. philips.com/news/con tent/file $152 \mathrm{htm}$ ) 